\title{
Does alcohol damage the adolescent brain? Neuroanatomical and neuropsychological consequences of adolescent drinking
}

This article was published in the following Dove Press journal:

Neuroscience and Neuroeconomics

9 December 2015

Number of times this article has been viewed

\author{
Rebekah L Fleming ${ }^{1,2}$ \\ 'Durham VA Medical Center, \\ ${ }^{2}$ Department of Psychiatry and \\ Behavioral Sciences, Duke University \\ Medical Center, Durham, NC, USA
}

\begin{abstract}
Alcohol drinking is a significant risk factor for morbidity and mortality in adolescents worldwide. Adolescents frequently binge drink, and this pattern of use is associated with poor school performance, injuries, violence, drug use, and a variety of poor psychosocial outcomes in adulthood. These associations have raised concerns that alcohol drinking may damage the adolescent brain and lead to impaired cognition and behavior. Similar to the neurotoxicity seen in adult alcoholics, magnetic resonance imaging studies of brain anatomy in adolescent drinkers have shown that alcohol disrupts the development of temporal and frontal cortices and myelinated fiber tracts throughout the brain. Although adult brains show some recovery with abstinence, at present, no studies have examined brain recovery in adolescents. Studies of neuropsychological function have found deficits in attention and visuospatial ability that show dose-dependent correlations with alcohol exposure and withdrawal symptoms, but visuospatial performance recovers with short-term abstinence. Differences in executive function and decisionmaking have also been found, but the available evidence suggests that these are not primarily the result of alcohol exposure; instead, they reflect premorbid factors that increase risk-taking and substance use. Nevertheless, alcohol drinking by adolescents remains an important concern because of the potential for brain injury in addition to the many negative consequences associated with acute intoxication.
\end{abstract}

Keywords: adolescence, binge drinking, alcohol, magnetic resonance imaging, neuropsychological function

\section{Introduction}

Alcohol drinking by adolescents is a major global health problem. In 2011, World Health Organization data were used to estimate the productive years of life lost due to premature mortality or disability in young people aged $15-24$ years. ${ }^{1}$ Alcohol use was the leading risk factor, accounting for $8 \%$ of lost productive years. Alcohol was found to be an especially significant risk factor for males from high-income countries, particularly the Americas and Europe.

In the USA, the National Survey on Drug Use and Health provides yearly data on the prevalence and patterns of alcohol use. For several decades, this survey has consistently shown rates of drinking that are very low in young teens, rise during adolescence, and peak in young adulthood. In 2013, rates of alcohol use in the past month were $2.1 \%$ for persons aged $12-13$ years, $22.7 \%$ for 16-17-year olds, and $69.3 \%$ for $21-25$-year olds. ${ }^{2}$ Rates of binge drinking, defined as five or more drinks on the same occasion in the past 30 days, were $13.1 \%$ for 16-17-year olds, while rates of heavy drinking, defined as five or more drinks per occasion on each of five or more days in the past
Correspondence: Rebekah L Fleming Durham VA Medical Center, Building 15, 508 Fulton Street, Durham, NC 27705, USA

Tel + I 91928604 I l ext 366I

Email rebekah.fleming@duke.edu
Neuroscience and Neuroeconomics 20I5:4 5I-60 (c) (i) (8) 2015 Fleming. This work is published by Dove Medical Press Limited, and licensed under Creative Commons Attribution - Non Commercial (unported, v3.0) License. The full terms of the License are available at http://creativecommons.org/licenses/by-nc/3.0/. Non-commercial uses of the work are permitted without any further permission how to request permission may be found at: http://www.dovepress.com/permissions.php 
month, were $2.7 \%$ (Table 1). Boys were more likely than girls to report binge or heavy drinking. Similar prevalence rates have been reported by a second US survey sponsored by the National Institute on Drug Abuse, but a third survey, the National Youth Risk Behavior Survey, reports higher prevalences, especially for young adolescents. ${ }^{3,4}$

The European School Survey Project on Alcohol and Other Drugs collected data on substance use among 15-16-year-old students from 36 European countries. National-level prevalences of alcohol use in the past month ranged from $17 \%$ (Iceland) to 69\% (Monaco) with an overall European-wide average of 57\%. ${ }^{5}$ National-level prevalences of binge drinking in the past month ranged from $13 \%$ (Iceland) to $56 \%$ (Malta) with an overall average of $39 \%$. Prevalences of drinking in the past 30 days mostly did not differ between boys and girls, but boys reported drinking 1/3 more alcohol in the last drinking episode. In the majority of European countries, boys reported binge drinking more frequently than girls. A 2015 study reported that drinking patterns in the People's Republic of China are similar to those in the USA and Europe. ${ }^{6}$ As in the studies from Europe and the USA, boys drank more than girls.

\section{Overview of adolescent drinking}

A significant amount of alcohol drinking is a normal part of the adolescent experience, especially for males. Adolescent patterns of drinking are a concern because adolescents are likely to engage in binge drinking, which results in higher blood alcohol levels, greater behavioral disinhibition and incoordination due to intoxication, and greater potential for alcohol-associated neurotoxicity. ${ }^{4,7}$ In addition to contributing to the leading causes of death in this age group, adolescent drinking is associated with a variety of adverse psychosocial outcomes, including mental illness, illegal substance use, earlier age of sexual debut and increased rates of regretted or unsafe sex, poor school performance or dropping out of school, increased risk of developing an alcohol or substance use disorder (SUD) as an adult, and downward social mobility.,4,7-11 These associations have led to the hypotheses that alcohol exposure interferes with adolescent brain development, leading to poor adjustment later in life.

\section{Mechanisms associated with adolescent brain maturation}

Adolescence is defined as the period of life between puberty and the attainment of a stable adult role in society. ${ }^{12}$ Puberty begins at the age of 10-13 years for girls and 12-15 years for boys, and sexual maturation is usually complete at the age of $\sim 17$ years. ${ }^{13}$ Studies of adolescent brain development have shown that puberty triggers a series of changes in brain structure, and maturation of some regions continues throughout adolescence and into young adulthood.

In humans, almost all neurons are present at birth, and the brain reaches its full weight at the age of $12-14$ years, the

Table I Drinking prevalences in the USA, Europe, and the People's Republic of China

\begin{tabular}{|c|c|c|c|c|c|c|c|c|c|c|c|}
\hline \multirow[t]{2}{*}{ Source } & \multirow[t]{2}{*}{ Location } & \multicolumn{10}{|c|}{ Age (Years) } \\
\hline & & 12 & 13 & 14 & 15 & 16 & 17 & 18 & 19 & 20 & $21-25$ \\
\hline \multicolumn{12}{|c|}{ Lifetime drinking prevalence (\%) } \\
\hline Johnston et $\mathrm{al}^{3}$ & USA & & 26.8 & & 49.3 & & 66.0 & & & & \\
\hline Hibell et $\mathrm{al}^{5}$ & Europe & & & & 87 & & & & & & \\
\hline Lu et al ${ }^{6}$ & People's Republic of China & & 39.8 & 52.0 & & 64.1 & 68.1 & & & & \\
\hline \multicolumn{12}{|c|}{ Alcohol use in past month (\%) } \\
\hline SAMHSA ${ }^{2}$ & USA & 2.1 & & 9.5 & & 22.7 & & 43.8 & & & 69.3 \\
\hline Johnston et $\mathrm{al}^{3}$ & USA & & 9.0 & & 23.5 & & 37.4 & & & & \\
\hline Miller et $\mathrm{al}^{4}$ & USA & 32.0 & & & 45.0 & & & 56.4 & & & \\
\hline Hibell et al ${ }^{5}$ & Europe & & & & 57 & & & & & & \\
\hline Lu et $\mathrm{al}^{6}$ & People's Republic of China & & 14.0 & 17.6 & & 27.5 & 27.4 & & & & \\
\hline \multicolumn{12}{|c|}{ Binge drinking in past month (\%) } \\
\hline SAMHSA $^{2}$ & USA & 0.8 & & 4.5 & & 13.1 & & 29.1 & & & 43.3 \\
\hline Johnston et $\mathrm{al}^{\mathrm{a}, 3}$ & USA & & 4.1 & & 12.6 & & 19.4 & & & & \\
\hline Miller et $\mathrm{al}^{4}$ & USA & 17.8 & & & 28.9 & & & 38.7 & & & \\
\hline Hibell et al ${ }^{5}$ & Europe & & & & 39 & & & & & & \\
\hline Lu et $\mathrm{al}^{6}$ & People's Republic of China & & 2.6 & 3.5 & & 7.6 & 8.1 & & & & \\
\hline \multicolumn{12}{|c|}{ Been drunk in last month (\%) } \\
\hline Johnston et $\mathrm{al}^{3}$ & USA & & 2.7 & & 11.2 & & 23.5 & & & & \\
\hline Hibell et $\mathrm{al}^{5}$ & Europe & & & & 17 & & & & & & \\
\hline Lu et $a l^{6}$ & People's Republic of China & & 8.3 & 12.1 & & 23.1 & 24.1 & & & & \\
\hline
\end{tabular}

Note: ${ }^{2}+$ drinks in a row in the last 2 weeks. 
beginning of adolescence. Instead of overall brain growth, maturation during adolescence consists of structural changes in the gray and white matter of specific brain regions. ${ }^{14-16}$ Postnatal brain maturation proceeds as a wave that starts in sensory and motor areas and moves anteriorly over time. Full maturation of association cortices in the frontal and temporal lobes occurs sometime during the age of late 20 's or early 30 's. ${ }^{17}$ In the dendrites of cortical neurons, synaptic maturation follows an inverted U-shaped function: the density of synapses increases until it reaches a peak; after the peak, synapses are lost as part of an experience-dependent pruning process that sculpts and refines the neural circuits within that region. ${ }^{16} \mathrm{~A}$ recent postmortem study examined synaptic spine development in prefrontal cortex (PFC) across the lifespan and showed that the spine density increased steadily throughout childhood and declined after puberty, suggesting that substantial refinement of PFC circuitry occurs during adolescence. ${ }^{18}$ Structural magnetic resonance imaging (MRI) studies have shown that cortical gray matter density and cortical thickness follow similar patterns, and the latest maturation occurs in PFC and temporal cortex..$^{19,20}$ Gray matter density in the striatum also declines during adolescence. ${ }^{21}$

The white matter of the brain is made up of myelin, a fatty substance that ensheathes the axons of neurons. Myelin functions as an electrical insulator and improves saltatory conduction of the action potential, allowing for quicker communication between brain regions. During development, myelination also occurs in a systematic sequence that progresses from inferior to superior brain regions and from posterior to anterior brain regions. ${ }^{22}$ Whole-brain white matter volumes increase throughout childhood, the rate of myelination accelerates at puberty, and the volumes continue to increase throughout adolescence and young adulthood to peak at $\sim 40$ years of age. ${ }^{15,17}$ Similar to the pattern seen for changes in gray matter, myelination of sensory and motor areas is completed during childhood, while the association areas, including the PFC and temporal cortex, are among the last to mature. White matter development in the cortico-cortical and cortico-limbic tracts also continues throughout adolescence. ${ }^{15}$ These increases in white matter volume are accompanied by progressive improvements in MRI measures of white matter integrity, fractional anisotropy (FA) and mean diffusivity, which reflect increasing microstructural organization of white matter tracts. ${ }^{15,16}$ Overall, more myelination and greater organization of fiber tracts result in more efficient communication among distant brain regions. Thus, proper white matter development in association cortices and tracts connecting distant brain regions may support the development of cognitive and emotional maturity in adolescents.

\section{Overview of brain adolescent development}

During adolescence, frontal and temporal association cortices undergo synaptic pruning and declines in gray matter that are accompanied by increased white matter volumes and greater organization of myelinated tracks that connect association regions to other areas of the brain. Disruption of these processes by alcohol exposure could lead to changes in the cognitive functions served by these brain regions.

\section{The effects of alcohol on adolescent brain development}

To determine if alcohol drinking during adolescence is associated with anatomical changes in the brain, structural MRI has been used to measure gray matter volumes and cortical thickness, white matter volumes, and white matter organization and integrity. Areas of particular interest for study were frontal and temporal cortical regions and associated white matter tracks, which are still developing during adolescence, along with areas that undergo damage in adult alcoholics, such as the hippocampus, cerebellum, and regions of the striatum. ${ }^{23}$ Several studies have also investigated whether there are differences in the effects of alcohol on boys and girls. As with all studies of the effects of substance use on the brain, these experiments are challenging to conduct and interpret because of the frequent presence of comorbid disorders that may contribute to apparent effects of alcohol drinking. Cross-sectional studies can be particularly subject to confounding, while longitudinal studies that assess changes using multiple measurements within individuals can avoid some of these artifacts. Therefore, cross-sectional and longitudinal studies are reviewed separately.

\section{Cross-sectional MRI studies of gray and white matter volumes}

An early study found that youth with an adolescent-onset alcohol use disorder (AUD) had significantly smaller bilateral hippocampal volumes than healthy controls. ${ }^{24}$ Hippocampal volumes correlated negatively with the duration of the AUD and positively with the age at the onset of AUD, suggesting a dose-dependent relationship between alcohol exposure and neurotoxicity. Total cortical gray and white matter volumes and amygdala volumes did not differ between groups. A later study that controlled for psychiatric comorbidity found that 
adolescents with AUD had significantly smaller left hippocampus volumes than healthy controls. ${ }^{25}$ However, no difference was found for right hippocampus, and there was no relationship between hippocampus volumes and alcohol use patterns. This finding of smaller left hippocampus volumes in adolescent drinkers was replicated in a later study that also controlled for conduct disorder. ${ }^{26}$ Here, smaller left hippocampal volumes were associated with more AUD symptoms. This study also reported a significant difference in right versus left hippocampal asymmetry in AUD subjects compared with controls.

De Bellis et $\mathrm{al}^{27}$ examined PFC, thalamic, and cerebellar volumes in adolescents with AUD and comorbid mental disorders. Subjects with AUD had smaller PFC gray and white matter volumes, and these volumes were significantly correlated with alcohol consumption. There were no sex differences in the effects of AUD on PFC volumes. There were no group differences in thalamic or cerebellar volumes, but there was a group by sex interaction indicating that males with AUD had smaller cerebellar volumes than controls with no effect of AUD in females.

Medina et $\mathrm{al}^{28}$ also compared adolescents with and without AUD. After controlling for sex and conduct disorder status, this study found no significant group differences in brain volume but did find a group by sex interaction. Girls with AUD had smaller total PFC volumes than same-sex controls, while AUD males had larger PFC volumes than controls. A similar pattern was observed for PFC white matter volumes. Interestingly, controlling for alcohol use and sex, this study found that adolescents with conduct disorder had significantly smaller PFC volumes. The authors did not find any correlations between lifetime alcohol use, alcohol withdrawal symptoms, or alcohol dependence symptoms and PFC volumes.

Squeglia et $\mathrm{al}^{29}$ reported sex differences in the effect of binge drinking on cortical thickness. In females, binge drinkers had significantly thicker cortices in two areas of PFC: the left frontal pole and the left pars orbitalis. Although females and males had similar estimated peak blood alcohol concentrations (BACs; 0.26 vs 0.28 , respectively), there was no effect of binge drinking on these brain regions in males. Male binge drinkers had significantly thinner cortices in left rostral anterior cingulate and left medial orbitofrontal cortex, with no effect in females in these brain regions. No group differences were found on a battery of seven neuropsychological tests. However, increased cortical thickness was associated with poorer cognitive performance for both females and males.
A large study of South African adolescents aged 12-16 years with an AUD, but without externalizing or other psychiatric disorders, found that compared to age- and sex-matched healthy controls, AUD adolescents showed decreased gray matter density in the left lateral frontal, temporal, and parietal lobes. ${ }^{30}$ This study also found two group by sex interactions on effects of AUD on subcortical volumes: AUD boys had smaller thalamus and putamen volumes compared with controls, whereas AUD girls had larger thalamus and putamen volumes. In contrast to other studies, ${ }^{24-26}$ no differences in hippocampus volumes were found. Interestingly, this study also showed deficits in cognition; AUD adolescents showed impaired verbal story memory and worse self-monitoring.

Lisdahl et $\mathrm{al}^{31}$ examined the effects of recent binge drinking (mean estimated BAC: 0.24) on cerebellar volumes in adolescents aged 16-19 years. Although no group differences in cerebellar gray or white matter were found, for binge drinkers, a higher maximum number of drinks per binge predicted smaller left and right cerebellar gray matter and smaller left cerebellar white matter, with a similar nonsignificant trend for right cerebellar white matter. No sex differences were found.

A second South African study ${ }^{32}$ addressed the question of whether childhood adversity could play a role in the effects of adolescent alcohol exposure on brain volumes. After covarying out childhood trauma, this study found a main effect of group on brain volumes in superior temporal gyrus, similar to their previous finding. ${ }^{30}$ There was no group effect of AUD on hippocampus volumes. However, in the total cohort, childhood trauma was linked to smaller total hippocampus volumes, with a stronger effect for left hippocampus. Childhood trauma score also positively correlated with lifetime alcohol intake. These results suggest that childhood adversity could be a confounding factor that contributes to apparent effects of AUD on brain structure, particularly for hippocampus volume.

\section{Cross-sectional MRI studies of white matter organization}

Three studies of white matter organization in adolescent drinkers have suggested that alcohol exposure disrupts the normal increases in white matter integrity that are characteristic of adolescent development. Jacobus et $\mathrm{al}^{33}$ found significantly lower FA, indicating less organization, in white matter regions within the superior corona radiata, inferior fronto-occipital fasciculus, superior and inferior longitudinal fasciculi, and middle cerebellar peduncle. Another study 
from the same research group found widespread reductions in FA in white matter pathways of the frontal, parietal, and temporal lobes as well as subcortical areas and cerebellum (mean estimated BAC: 0.24).$^{34}$ Reductions in FA were found by a second group using a different population, youth involved with the juvenile justice system. ${ }^{35}$ Compared to low-scoring controls, adolescents with a high Alcohol Use Disorders Identification Test (AUDIT) score had significantly lower FA in regions from the right and left posterior corona radiata and right superior longitudinal fasciculus. One cluster in the right anterior corona radiata showed higher FA in the high AUDIT group.

Two other studies have also shown increased FA, indicating better white matter organization, in adolescents with AUD. De Bellis et $\mathrm{al}^{36}$ found increased FA in the rostrum of the corpus callosum, near the isthmus region of the anterior cingulate. In addition, the developmental trajectory of FA was disturbed in the AUD group; controls showed the expected pattern of positive correlation of FA with age, but in AUD subjects the effect was reversed and FA was negatively correlated with age, which is consistent with a neurotoxic effect of alcohol on myelination. This study also found a sex by group interaction; girls with AUD had lower FA in the posterior midbody of the corpus callosum, which is similar to the reduced FA in the three studies described earlier. However, a large South African study failed to find evidence of a neurotoxic effect on white matter development. ${ }^{37}$ In this study, AUD subjects did not have a comorbid substance abuse or externalizing disorders, and the authors controlled for childhood adversity. AUD subjects had higher FA in two tracts of the limbic system, the fornix and stria terminalis. No sex differences were found. The increased FA was not associated with any measure of alcohol use. Neuropsychological testing found impairment of verbal story memory and self-monitoring in AUD subjects. In contrast with most other studies, no regions of reduced FA were found in AUD subjects. The authors suggested that greater prevalence of trauma and lower SES in their sample could explain the discrepancy between this and other studies. In studies that did not adequately control for externalizing or conduct disorders, disruptions of myelination associated with a behavior disorder could also have contributed to differences in FA. ${ }^{38}$

\section{Longitudinal MRI studies of gray and white matter}

A longitudinal study of adolescents aged 14-19 years at baseline who initiated alcohol use during a 2-year study duration found that initiators had greater loss of cortical thickness in the right middle frontal gyrus of the PFC. ${ }^{39}$ Compared to nondrinking controls, initiators also showed reduced gains in white matter in right precentral gyrus (motor cortex), lingual gyrus (parietal cortex, visual association cortex), temporal gyrus, and anterior cingulate cortex. Initiators also had decreases in FA in the left caudate/thalamic region and right mid-temporal/polar-temporal region of the inferior fronto-occipital fasciculus. An important aspect of this study is that it examined subclinical levels of drinking; the initiators reported using alcohol 3.9 times per month, with an average drinks per occasion of 5.4, and an average total drinks per month of 22.3.

Three other longitudinal studies from Susan Tapert's group in San Diego, CA, examined youth who transitioned to heavy or binge drinking during adolescence. The first study ${ }^{40}$ found that in no-drinking controls, FA increased over time in 15 regions of interest throughout the brain, reflecting the expected developmental trajectory of improved white matter organization with age. However, adolescents who escalated to heavy drinking showed declines in white matter integrity from baseline to follow-up. At the 3-year follow-up, there were also group differences in FA between binge drinkers and controls, with drinkers showing significantly poorer white matter integrity. The second study ${ }^{41}$ found that at baseline, when all subject were nondrinkers, subjects who later became heavy drinkers had smaller volumes in regions of left cingulate and frontal cortex and less right cerebellar white matter. At the 3-year follow-up, those who became heavy drinkers showed significantly more volume reductions in left diencephalon, temporal lobe, caudate, and brainstem. For heavy drinkers, more lifetime alcohol use was correlated with greater volume reductions in the left caudate. The third and largest study ${ }^{42}$ more thoroughly examined brain developmental trajectories in adolescents who initiated heavy drinking. These drinkers averaged 210 lifetime alcohol use occasions, 9.69 drinking days per month during the 3 months before follow-up, 7.56 peak drinks per occasion, and an estimated peak BAC of 0.21 , which is consistent with binge drinking on Friday and Saturday of every weekend. Controls (non- or light drinkers) showed brain changes consistent with normal adolescent development: gray matter volumes decreased in multiple cortical regions, and all three white matter regions studied (corpus callosum, central white matter, and pons) showed increases in volume at follow-up. In contrast, adolescents who transitioned to heavy drinking deviated from the normal trajectory: cortical gray matter loss was accelerated in lateral frontal and temporal cortices, and white matter growth was slowed. In contrast to smaller 
studies showing sex differences, ${ }^{28,29}$ male and female heavy drinkers showed similar patterns of altered gray and white matter development. However, it is important to note that this study found several group differences between controls and heavy drinkers that could act as confounds: heavy drinkers were older, further along in pubertal development at baseline, and had significantly higher scores for depression and externalizing behaviors.

\section{Summary of the effects of alcohol drinking on gray and white matter development}

Of the changes seen in gray matter, the strongest cumulative evidence is for decreases in cortical volume or thickness in the temporal lobe. These changes were found in two relatively large cross-sectional studies that controlled for many confounds $\mathrm{s}^{30,32}$ and in three longitudinal studies..$^{39,41,42}$ These four studies represent three sample populations from two different parts of the world. Decreases in volume or thickness in frontal regions of the brain, including PFC, are next in strength of evidence; these were found in four cross-sectional ${ }^{27-30}$ and two longitudinal studies ${ }^{39,42}$ from three populations. Two studies of sex differences showed opposite effects of drinking on PFC development in girls. ${ }^{28,29}$ In spite of the larger number of studies, the evidence for effects on frontal cortex is somewhat weakened by possible associations with confounds such as conduct disorder ${ }^{28}$ or childhood adversity. ${ }^{32}$ The finding of reduced frontal cortex volumes at baseline in a longitudinal study ${ }^{41}$ also suggests that dysregulation of frontal cortex development may be a predisposing factor for alcohol drinking. ${ }^{43,44}$ However, accelerated loss of gray matter in a longitudinal study ${ }^{42}$ suggests that even if predrinking differences are present, alcohol may cause some neurotoxicity in frontal cortex. In hippocampus, there is abundant evidence for damage in adult alcoholics, but the evidence for an effect of adolescent drinking on this region is equivocal. Decreases in hippocampus volumes were found in three small crosssectional studies ${ }^{24,25,45}$ but were not replicated by two larger studies, ${ }^{30,32}$ one of which identified a potential confounding association of reduced hippocampus volume with childhood adversity. ${ }^{32}$ Studies of gray matter in cerebellum and subcortical regions are suggestive of possible effects of alcohol, but the evidence is still preliminary. Sex differences identified in small studies ${ }^{28,29}$ have not been replicated by larger studies, ${ }^{30,42}$ so at present, there is limited evidence for greater vulnerability in either girls or boys.

In contrast with the mixed evidence for effects of adolescent drinking on gray matter, the majority of studies reviewed present evidence for neurotoxic effects of alcohol on developing myelinated pathways, ${ }^{33-35,39,40,42}$ which is consistent with the degradation of white matter tracts seen in adult alcoholics. (The exception to this pattern is the South African study that found no decreased areas of $\mathrm{FA}^{37}$ ). Although the potential for neurotoxic effects on white matter is a significant concern, the fact that adult alcoholics show recovery of white matter integrity after 1 year of abstinence ${ }^{46}$ may be reassuring because it suggests the possibility that the adolescent brain could recover from damage if the youth stop drinking.

\section{Consequence of these effects on adolescent behavior and cognition}

Since the early 1900s, adolescence has been described as a developmental period characterized by cognitive impulsivity, risk-seeking, emotional intensity and lability, and social reorientation. ${ }^{14}$ The dyadic systems model is a conceptual framework that describes changes in brain function that may account for these typical adolescent behaviors. According to this model, the choice to inhibit risky, immediately rewarding behavior in favor of long-term goals is determined by the balance between two opposing processes: incentive motivation and executive function..$^{12,44,47}$ The incentive motivation system is served by the striatum as well as the medial and orbital frontal cortices, whereas executive function, or cognitive control, is served by lateral PFC and temporal cortex. ${ }^{48,49}$ Adolescent impulsiveness and risk-taking occur because puberty triggers a peak in striatum-driven incentive motivation that occurs at $\sim 15-16$ years of age, before the cognitive control system has reached full maturity. ${ }^{12}$ This peak in responsiveness of the striatal dopamine system may also explain adolescent experimentation with alcohol and drugs. ${ }^{50}$ However, although this model has been widely applied to explain adolescent behavior, it has been critiqued by others who note that higher rates of substance use, unintentional injuries, and death by violence occur in young adulthood when the cognitive control system is almost completely mature. ${ }^{13,47,51}$ Some authors suggest that excessive risk-taking and substance use are not significant features of normal adolescence; instead, they occur in a subset of teens who have a history of behavioral disinhibition and whose brains may not be developing typically. ${ }^{44,47}$ This possibility is important to consider when examining the putative effects of adolescent drinking on brain function because it suggests that the heaviest drinkers may have neuropsychological differences that preceded alcohol exposure. Indeed, a family history of alcoholism is a major risk factor for developing an AUD, and studies of family history positive individuals strongly suggest that deficits in executive function and affective regulation represent a cognitive endophenotype that confers increased 
risk for early substance use. ${ }^{52,53}$ Many studies of adolescent drinkers show increased rates of externalizing and conduct disorders, which indicate problems with executive function. ${ }^{44}$ Longitudinal studies with a preexposure baseline can help to determine what comes first: disinhibition or drinking, but if continuing brain development is abnormal in risk-taking and/ or family history positive adolescents, ${ }^{38}$ cognitive differences could be misattributed to alcohol neurotoxicity.

Evidence from adults suggests that prolonged alcohol abuse leads to widespread deficits in cognition. A recent meta-analysis of 62 studies $^{54}$ reported that after short-term abstinence, adult alcoholics showed performance deficits in eleven cognitive domains: verbal fluency/language, speed of processing, working memory, attention, problem solving/ executive functions, inhibition/impulsivity, verbal learning, verbal memory, visual learning, visual memory, and visuospatial abilities. Effects were mostly of moderate size, and Cohen's $d$ ranged from 0.4 (visual learning) to 0.7 (attention). In contrast, studies of adolescents have found deficits in some of these domains, suggesting more selective effects on alcohol exposure on cognitive ability. For example, an early study ${ }^{55}$ compared alcohol-dependent adolescents to a community sample matched for demographic characteristics. Neuropsychological function was assessed using a battery of eight tests performed after a minimum of 3 weeks of abstinence. Poorer performance was found on tests in the domains of language, memory, and visuospatial cognition, with the most consistent deficits found in the language domain. The subjects of this study were adolescents with a history of substantial alcohol exposure; at ages 15-16, the mean number of times they had drunk during their lifetime was 753 , the mean drinking days per month over the 3 months before the study was 18.76, and the mean drinks per day was five. For comparison, according to the National Survey on Drug Use and Health, in 2013, an estimated 2.8\% of youth aged 12-17 years had an AUD. In addition, 2.7\% of 16-17-year olds in the USA reported heavy drinking, which accounts for $12 \%$ of those who drank any alcohol. ${ }^{2}$

Another study from the same research group ${ }^{56}$ examined the effects of continued alcohol and/or drug use in 115 adolescents who received impatient treatment for an AUD or SUD (an estimated 5.7\% of youth had an alcohol or drug use disorder in 20132). Subjects were followed for 4 years, and continued use of substances after treatment was associated with poorer functioning on tests of attention (Cohen's $d=0.53$ for abusers with no recent use). No group differences were found for the visuospatial, memory, or intrusion domains. However, multiple regression analysis indicated that the number of withdrawal symptoms reported for the 3 months preceding the 4-year follow-up predicted poorer visuospatial ability $\left(R^{2}=0.17\right)$. Further evaluation of the effects of withdrawal revealed that reporting symptoms during the 3 months preceding the follow-up was associated with poorer performance in the visuospatial, attention, and intrusion domains. These subjects were followed for another 4 years and compared to a community sample of adolescents without SUDs. ${ }^{57}$ Participants with a history of SUD at intake or during the follow-up period performed worse than controls in the attention domain (Cohen's $d=0.54$ ). Hierarchical regression analyses were used to estimate the contributions of different substances to declines in cognitive performance, and cumulative alcohol use over the 8 years of the study predicted poorer attention functioning with a small effect size $\left(f^{2}=0.3\right)$. Subjects reporting any withdrawal during the 8 years had worse performance on tests of attention and visuospatial ability, and cumulative substance withdrawal predicted attention and visuospatial function at year 8, with alcohol withdrawal specifically predicting visuospatial performance. At a 10-year follow-up, cumulative alcohol use predicted poorer visuospatial performance, but alcohol withdrawal was no longer associated with poorer visuospatial ability. ${ }^{45}$

A recent study ${ }^{58}$ investigated neuropsychological deficits and recovery in heavy drinking adolescents. The criteria for heavy drinking included $\geq 50$ lifetime drinking episodes, $\geq 1$ heavy drinking episodes in the last month, and importantly, $\geq 1$ withdrawal symptom in the past 2 weeks. Drinkers were matched to controls for measures of academic performance before the initiation of drinking. Subjects were administered three batteries of tests over a 4-week abstinence period. At the beginning of the study, with an average abstinence of 5.5 days, heavy drinkers performed worse than controls on tests of prospective memory, cognitive switching, inhibition, verbal memory, visuospatial construction, and language achievement, with effect sizes ranging from 0.59 to 1.04 . Scores improved over time for both groups, but heavy drinkers improved more rapidly on some tasks, possibly representing partial recovery from the effects of alcohol exposure, similar to that reported for adults. ${ }^{54}$ However, only the scores for visuospatial construction improved enough to bring heavy drinkers to control levels after 4 weeks of abstinence. The effect of heavy drinking on visuospatial function is supported by another study of adolescents who transitioned to heavy drinking; in girls, past year drinking days predicted worsening visuospatial performance. ${ }^{59}$ Another recent study ${ }^{60}$ compared heavy drinking adolescents, heavy drinkers who also used marijuana, marijuana users only, and controls who had been matched for family history of substance dependence and academic performance. After 1 month of withdrawal, 
heavy drinkers had worse performance than controls on tests of cognitive switching (Cohen's $d=0.88$ ) and verbal memory. More alcohol withdrawal symptoms were associated with poorer cognitive switching. In contrast with other studies of adolescent drinkers, ${ }^{45,59}$ but consistent with the recovery seen previously, ${ }^{58}$ this study did not find significant group differences for tests of visuospatial construction. However, the authors did find a significant correlation between alcohol withdrawal symptoms and worse visuospatial performance.

Two studies have examined affective decision-making in adolescent binge drinkers using the Iowa Gambling Test (IGT), which simulates real-world risky decision-making. Binge drinkers showed significantly worse performance on this task, and additional testing using a variant of the IGT suggested that this poor performance was due to hypersensitivity to reward that made high-payoff risky choices more attractive to binge drinkers. ${ }^{61}$ Furthermore, poor performance on the IGT predicted binge drinking 1 year later, suggesting that impaired affective decision-making may be a factor that predisposes an individual toward binge drinking. ${ }^{62}$ Similarly, a large-scale modeling study found that higher delay discounting is associated with an increased likelihood of binge drinking at the age of 14 years. ${ }^{43}$

\section{Summary of the effects of alcohol on adolescent cognition}

Studies of adolescent heavy drinkers or those with AUD/SUD have shown that alcohol exposure is linked to deficits in language ability, visuospatial cognition, and executive function, as indicated by poorer performance in attention, memory, cognitive switching, and inhibition tasks. Adolescent binge drinkers also show deficits in affective decision-making, which suggest changes in the incentive motivation system. ${ }^{43,44,49}$ Cumulative alcohol exposure and withdrawal symptoms have been repeatedly associated with worse impairments of attention and visuospatial ability, and these dose-dependent relationships suggest neurotoxicity as a possible cause of these deficits. Modest effect sizes for group differences suggest that heavy alcohol drinking does not cause clinical impairment, even in adolescents with AUD; rather, these are subtle differences that might contribute to the poor psychosocial outcomes that are widely associated with adolescent drinking. ${ }^{63}$ Longitudinal studies provide support for the hypothesis that alcohol neurotoxicity causes declines in visuospatial performance, which is consistent with impaired development of the temporal lobes, but this can recover with abstinence..$^{58,59}$

The relationships between alcohol drinking and differences in executive function and incentive motivation are probably more complex. Behavioral disinhibition during childhood has been identified as an important predictor of and risk factor for adolescent-onset addiction ${ }^{44}$ and adolescent binge drinking. ${ }^{43,62}$ Therefore, differences in memory, attention, cognitive switching, and risky decision-making could reflect predisposing factors that contribute to the development of heavy alcohol use. ${ }^{44,47}$ In spite of the presence of premorbid factors, the evidence that alcohol interferes with the development of frontal cortex, temporal cortex, and associated myelinated tracks suggests that neurotoxic injury may impair further development of executive function, leading to a feed-forward loop in which individuals with a predisposition for behavioral problems and substance use become even less capable of self-regulation due to neurotoxic damage. However, a recent study that directly tested this feed-forward hypothesis found no evidence that alcohol drinking predicted changes in impulsivity. ${ }^{64}$ Therefore, the role of drinking in causing executive dysfunction remains uncertain.

\section{Conclusion}

Alcohol drinking is a significant risk factor for morbidity and mortality in adolescents worldwide, especially in males. ${ }^{1}$ Excessive drinking is also associated with poor school performance, drug use, and a variety of poor psychosocial outcomes in adulthood, including greater risk of an AUD or SUD. ${ }^{8-11}$ These associations have raised concerns that alcohol drinking may damage the adolescent brain and lead to impaired cognition and decision-making. MRI studies of adolescent drinkers have shown that alcohol exposure disrupts the development of temporal $1^{30,32,39,41,42}$ and frontal ${ }^{27-30,39,42}$ cortices and myelinated fiber tracks throughout the brain. ${ }^{33-35,39,40,42}$ It is not known if these effects reverse if drinking stops. Studies of cognition have found modest deficits in attention and visuospatial ability, and visuospatial performance recovers with abstinence. ${ }^{58}$ Differences in executive function and affective decision-making have also been found, but careful examination of the evidence suggests that these differences are not primarily the result of alcohol exposure; instead, they reflect premorbid factors that predispose an individual toward risk-taking and substance use. ${ }^{44,47}$ Therefore, although anatomical studies suggest that heavy alcohol exposure can disrupt adolescent brain development, the relationship between altered brain structure and psychosocial impairment is still uncertain. Longitudinal studies of the effects of drinking on adolescent cognition have usually observed subtle impairments, which may reflect an adaptive capacity of the adolescent brain that allows it to compensate for the effects of alcohol exposure. ${ }^{65,66}$ Preexisting neurocognitive differences, 
not alcohol-induced brain damage, may account for many of the behavioral difficulties observed in binge drinking adolescents. Nevertheless, alcohol drinking by adolescents remains an important concern because of the potential for brain injury in addition to the many negative consequences associated with acute intoxication.

\section{Acknowledgment}

This work was supported by Merit Review Award \# I01BX002128 from the United States (US) Department of Veterans Affairs Biomedical Laboratory Research and Development Service.

\section{Disclosure}

The author reports no conflicts of interest in this work. The views expressed in this article are those of the author and do not necessarily reflect the position or policy of the United States Department of Veterans Affairs or the United States Government.

\section{References}

1. Gore FM, Bloem PJ, Patton GC, et al. Global burden of disease in young people aged 10-24 years: a systematic analysis. Lancet. 2011; 377(9783):2093-2102.

2. Substance Abuse and Mental Health Services Administration. Results from the 2013 national survey on drug use and health: summary of national findings, NSDUH Series H-48, HHS Publication No (SMA) 14-4863. Rockville, MD: Substance Abuse and Mental Health Services Administration, 2014.

3. Johnston LD, O'Malley PM, Miech RA, Bachman JG, Schulenberg JE. Monitoring the Future national Survey Results on Drug Use: 1975-2014: Overview, Key Findings on Adolescent Drug Use. Ann Arbor, MI: Institute for Social Research, The University of Michigan; 2015.

4. Miller JW, Naimi TS, Brewer RD, Jones SE. Binge drinking and associated health risk behaviors among high school students. Pediatrics. 2007;119(1):76-85.

5. Hibell B, Guttormsson U, Ahlström S, et al. The 2011 ESPAD ReportSubstance Use Among Students in 36 European Countries. Stockholm, Sweden: The Swedish Council for Information on Alcohol and other Drugs; 2012

6. Lu S, Du S, Hu X, et al. Drinking patterns and the association between socio-demographic factors and adolescents' alcohol use in three metropolises in China. Int J Environ Res Public Health. 2015;12(2): 2037-2053.

7. Zeigler DW, Wang CC, Yoast RA, et al; Council on Scientific Affairs; American Medical Association. The neurocognitive effects of alcohol on adolescents and college students. Prev Med. 2005;40(1):23-32.

8. Bonomo Y, Coffey C, Wolfe R, Lynskey M, Bowes G, Patton G. Adverse outcomes of alcohol use in adolescents. Addiction. 2001; 96(10):1485-1496.

9. Rose RJ, Winter T, Viken RJ, Kaprio J. Adolescent alcohol abuse and adverse adult outcomes: evaluating confounds with drinking-discordant twins. Alcohol Clin Exp Res. 2014;38(8):2314-2321.

10. Kuntsche E, Rossow I, Simons-Morton B, Bogt TT, Kokkevi A, Godeau E. Not early drinking but early drunkenness is a risk factor for problem behaviors among adolescents from 38 European and North American countries. Alcohol Clin Exp Res. 2013;37(2):308-314.
11. Anderson KG, Ramo DE, Cummins KM, Brown SA. Alcohol and drug involvement after adolescent treatment and functioning during emerging adulthood. Drug Alcohol Depend. 2010;107(2-3):171-181.

12. Steinberg L. A social neuroscience perspective on adolescent risk-taking. Dev Rev. 2008;28(1):78-106.

13. Males M. Does the adolescent brain make risk taking inevitable? A skeptical appraisal. J Adolesc Res. 2009;24(1):3-20.

14. Arnett JJ. G Stanley Hall's adolescence: brilliance and nonsense. Hist Psychol. 2006;9(3):186-197.

15. Elofson J, Gongvatana W, Carey KB. Alcohol use and cerebral white matter compromise in adolescence. Addict Behav. 2013;38(7): 2295-2305.

16. Blakemore SJ. Imaging brain development: the adolescent brain. Neuroimage. 2012;61(2):397-406.

17. Sowell ER, Peterson BS, Thompson PM, Welcome SE, Henkenius AL, Toga AW. Mapping cortical change across the human life span. Nat Neurosci. 2003;6(3):309-315.

18. Petanjek Z, Judaš M, Šimic G, et al. Extraordinary neoteny of synaptic spines in the human prefrontal cortex. Proc Natl Acad Sci U SA. 2011; 108(32):13281-13286.

19. Gogtay N, Giedd JN, Lusk L, et al. Dynamic mapping of human cortical development during childhood through early adulthood. Proc Natl Acad Sci U S A. 2004;101(21):8174-8179.

20. Shaw P, Kabani NJ, Lerch JP, et al. Neurodevelopmental trajectories of the human cerebral cortex. J Neurosci. 2008;28(14):3586-3594.

21. Sowell ER, Thompson PM, Holmes CJ, Jernigan TL, Toga AW. In vivo evidence for post-adolescent brain maturation in frontal and striatal regions. Nat Neurosci. 1999;2(10):859-861.

22. Sowell ER, Thompson PM, Toga AW. Mapping changes in the human cortex throughout the span of life. Neuroscientist. 2004;10(4): 372-392.

23. Rosenbloom MJ, Pfefferbaum A. Magnetic resonance imaging of the living brain: evidence for brain degeneration among alcoholics and recovery with abstinence. Alcohol Res Health. 2008;31(4):362-376.

24. De Bellis MD, Clark DB, Beers SR, et al. Hippocampal volume in adolescent-onset alcohol use disorders. Am J Psychiatry. 2000;157(5): 737-744.

25. Nagel BJ, Schweinsburg AD, Phan V, Tapert SF. Reduced hippocampal volume among adolescents with alcohol use disorders without psychiatric comorbidity. Psychiatry Res. 2005;139(3):181-190.

26. Medina KL, Schweinsburg AD, Cohen-Zion M, Nagel BJ, Tapert SF. Effects of alcohol and combined marijuana and alcohol use during adolescence on hippocampal volume and asymmetry. Neurotoxicol Teratol. 2007;29(1):141-152.

27. De Bellis MD, Narasimhan A, Thatcher DL, Keshavan MS, Soloff P, Clark DB. Prefrontal cortex, thalamus, and cerebellar volumes in adolescents and young adults with adolescent-onset alcohol use disorders and comorbid mental disorders. Alcohol Clin Exp Res. 2005;29(9):1590-1600.

28. Medina KL, McQueeny T, Nagel BJ, Hanson KL, Schweinsburg AD, Tapert SF. Prefrontal cortex volumes in adolescents with alcohol use disorders: unique gender effects. Alcohol Clin Exp Res. 2008;32(3): 386-394.

29. Squeglia LM, Sorg SF, Schweinsburg AD, Wetherill RR, Pulido C, Tapert SF. Binge drinking differentially affects adolescent male and female brain morphometry. Psychopharmacology. 2012;220(3): 529-539.

30. Fein G, Greenstein D, Cardenas VA, et al. Cortical and subcortical volumes in adolescents with alcohol dependence but without substance or psychiatric comorbidities. Psychiatry Res. 2013;214(1):1-8.

31. Lisdahl KM, Thayer R, Squeglia LM, McQueeny TM, Tapert SF. Recent binge drinking predicts smaller cerebellar volumes in adolescents. Psychiatry Res. 2013;211(1):17-23.

32. Brooks SJ, Dalvie S, Cuzen NL, Cardenas V, Fein G, Stein DJ. Childhood adversity is linked to differential brain volumes in adolescents with alcohol use disorder: a voxel-based morphometry study. Metab Brain Dis. 2014;29(2):311-321. 
33. Jacobus J, McQueeny T, Bava S, et al. White matter integrity in adolescents with histories of marijuana use and binge drinking. Neurotoxicol Teratol. 2009;31(6):349-355.

34. McQueeny T, Schweinsburg BC, Schweinsburg AD, et al. Altered white matter integrity in adolescent binge drinkers. Alcohol Clin Exp Res. 2009;33(7):1278-1285.

35. Thayer RE, Callahan TJ, Weiland BJ, Hutchison KE, Bryan AD. Associations between fractional anisotropy and problematic alcohol use in juvenile justice-involved adolescents. Am J Drug Alcohol Abuse. 2013;39(6):365-371.

36. De Bellis MD, Van Voorhees E, Hooper SR, et al. Diffusion tensor measures of the corpus callosum in adolescents with adolescent onset alcohol use disorders. Alcohol Clin Exp Res. 2008;32(3):395-404.

37. Cardenas VA, Greenstein D, Fouche JP, et al. Not lesser but greater fractional anisotropy in adolescents with alcohol use disorders. Neuroimage Clin. 2013;2:804-809.

38. Hummer TA, Wang Y, Kronenberger WG, Dunn DW, Mathews VP. The relationship of brain structure to age and executive functioning in adolescent disruptive behavior disorder. Psychiatry Res. 2015;231(3): 210-217.

39. Luciana M, Collins PF, Muetzel RL, Lim KO. Effects of alcohol use initiation on brain structure in typically developing adolescents. Am J Drug Alcohol Abuse. 2013;39(6):345-355.

40. Jacobus J, Squeglia LM, Bava S, Tapert SF. White matter characterization of adolescent binge drinking with and without co-occurring marijuana use: a 3-year investigation. Psychiatry Res. 2013;214(3): 374-381.

41. Squeglia LM, Rinker DA, Bartsch H, et al. Brain volume reductions in adolescent heavy drinkers. Dev Cogn Neurosci. 2014;9:117-125.

42. Squeglia LM, Tapert SF, Sullivan EV, et al. Brain development in heavy-drinking adolescents. Am J Psychiatry. 2015;172(6):531-542.

43. Whelan R, Watts R, Orr CA, et al; IMAGEN Consortium. Neuropsychosocial profiles of current and future adolescent alcohol misusers. Nature. 2014;512(7513):185-189.

44. Iacono WG, Malone SM, McGue M. Behavioral disinhibition and the development of early-onset addiction: common and specific influences. Annu Rev Clin Psychol. 2008;4:325-348.

45. Hanson KL, Medina KL, Padula CB, Tapert SF, Brown SA. Impact of adolescent alcohol and drug use on neuropsychological functioning in young adulthood: 10-year outcomes. J Child Adolesc Subst Abuse. 2011;20(2):135-154.

46. Alhassoon OM, Sorg SF, Taylor MJ, et al. Callosal white matter microstructural recovery in abstinent alcoholics: a longitudinal diffusion tensor imaging study. Alcohol Clin Exp Res. 2012;36(11): 1922-1931.

47. Bjork JM, Pardini DA. Who are those "risk-taking adolescents"? Individual differences in developmental neuroimaging research. Dev Cogn Neurosci. 2015;11:56-64.

48. Ernst M. The triadic model perspective for the study of adolescent motivated behavior. Brain Cogn. 2014;89:104-111.

49. McClure SM, Bickel WK. A dual-systems perspective on addiction: contributions from neuroimaging and cognitive training. Ann NY Acad Sci. 2014;1327:62-78.
50. Urosevic S, Collins P, Muetzel R, Lim K, Luciana M. Longitudinal changes in behavioral approach system sensitivity and brain structures involved in reward processing during adolescence. Dev Psychol. 2012;48(5):1488-1500.

51. Willoughby T, Good M, Adachi PJ, Hamza C, Tavernier R. Examining the link between adolescent brain development and risk taking from a social-developmental perspective. Brain Cogn. 2013;83(3):315-323.

52. Tessner KD, Hill SY. Neural circuitry associated with risk for alcohol use disorders. Neuropsychol Rev. 2010;20(1):1-20.

53. Cservenka A, Alarcon G, Jones SA, Nagel BJ. Advances in human neuroconnectivity research: applications for understanding familial history risk for alcoholism. Alcohol Res. 2015;37(1):89-95.

54. Stavro K, Pelletier J, Potvin S. Widespread and sustained cognitive deficits in alcoholism: a meta-analysis. Addict Biol. 2013;18(2):203-213.

55. Brown SA, Tapert SF, Granholm E, Delis DC. Neurocognitive functioning of adolescents: effects of protracted alcohol use. Alcohol Clin Exp Res. 2000;24(2):164-171.

56. Tapert SF, Brown SA. Neuropsychological correlates of adolescent substance abuse: four-year outcomes. J Int Neuropsychol Soc. 1999;5(6):481-493.

57. Tapert SF, Granholm E, Leedy NG, Brown SA. Substance use and withdrawal: neuropsychological functioning over 8 years in youth. J Int Neuropsychol Soc. 2002;8(7):873-883.

58. Winward JL, Hanson KL, Bekman NM, Tapert SF, Brown SA. Adolescent heavy episodic drinking: neurocognitive functioning during early abstinence. J Int Neuropsychol Soc. 2014;20(2):218-229.

59. Squeglia LM, Spadoni AD, Infante MA, Myers MG, Tapert SF. Initiating moderate to heavy alcohol use predicts changes in neuropsychological functioning for adolescent girls and boys. Psychol Addict Behav. 2009;23(4):715-722.

60. Winward JL, Hanson KL, Tapert SF, Brown SA. Heavy alcohol use, marijuana use, and concomitant use by adolescents are associated with unique and shared cognitive decrements. J Int Neuropsychol Soc. 2014;20(8):784-795

61. Johnson CA, Xiao L, Palmer P, et al. Affective decision-making deficits, linked to a dysfunctional ventromedial prefrontal cortex, revealed in 10th grade Chinese adolescent binge drinkers. Neuropsychologia. 2008;46(2):714-726

62. Xiao L, Bechara A, Grenard LJ, et al. Affective decision-making predictive of Chinese adolescent drinking behaviors. J Int Neuropsychol Soc. 2009;15(4):547-557.

63. Hanson KL, Cummins K, Tapert SF, Brown SA. Changes in neuropsychological functioning over 10 years following adolescent substance abuse treatment. Psychol Addict Behav. 2011;25(1):127-142.

64. Fernie G, Peeters M, Gullo MJ, et al. Multiple behavioural impulsivity tasks predict prospective alcohol involvement in adolescents. Addiction. 2013;108(11):1916-1923.

65. Wiers RW, Boelema SR, Nikolaou K, Gladwin TE. On the development of implicit and control processes in relation to substance use in adolescence. Curr Addict Rep. 2015;2(2):141-155.

66. Crone EA, Dahl RE. Understanding adolescence as a period of socialaffective engagement and goal flexibility. Nat Rev Neurosci. 2012;13(9): 636-650.
Neuroscience and Neuroeconomics

\section{Publish your work in this journal}

Neuroscience and Neuroeconomics is an international, peer-reviewed, open access journal focusing on the identification of brain structures and measurement of neural activity related to behavior, behavioral predictions, and decision making in health and disease. The manuscript
Dovepress

management system is completely online and includes a very quick and fair peer-review system. Visit http://www.dovepress.com/testimonials php to read real quotes from published authors. 